\title{
QUANTIZATION OF SYMPLECTIC DYNAMICAL $r$-MATRICES AND THE QUANTUM COMPOSITION FORMULA
}

\author{
ANTON ALEKSEEV AND DAMIEN CALAQUE
}

\begin{abstract}
In this paper we quantize symplectic dynamical $r$-matrices over a possibly nonabelian base. The proof is based on the fact that the existence of a star-product with a nice property (called strong invariance) is sufficient for the existence of a quantization. We also classify such quantizations and prove a quantum analogue of the classical composition formula for coboundary dynamical $r$-matrices.
\end{abstract}

\section{INTRODUCTION}

Let $\mathfrak{h} \subset \mathfrak{g}$ be an inclusion of Lie algebras and $H \subset G$ the corresponding inclusion of Lie groups. Let $U \subset \mathfrak{h}^{*}$ be an invariant open subset and let $Z \in\left(\wedge^{3} \mathfrak{g}\right)^{\mathfrak{g}}$. A (coboundary) dynamical $r$-matrix is a h-equivariant map $r: U \rightarrow \wedge^{2} \mathfrak{g}$ satisfying the (modified) classical dynamical Yang-Baxter equation

$$
\frac{1}{2}[r(\lambda), r(\lambda)]-\sum_{i} h_{i} \wedge \frac{\partial r}{\partial \lambda^{i}}(\lambda)=Z \quad(\lambda \in U),
$$

where $\left(h_{i}\right)_{i}$ and $\left(\lambda^{i}\right)_{i}$ are dual bases of $\mathfrak{h}$ and $\mathfrak{h}^{*}$, respectively. Then (following [14, 6])

$$
\pi_{r}:=\pi_{l i n}+\sum_{i} \frac{\partial}{\partial \lambda^{i}} \wedge \overrightarrow{h_{i}}+\overrightarrow{r(\lambda)},
$$

together with $Z$, defines a $H$-invariant (g-)quasi-Poisson structure on $M=U \times G$. Here $\pi_{l i n}$ is the linear Poisson structure on $U \subset \mathfrak{h}^{*}$.

Such a dynamical $r$-matrix is called symplectic if the $\mathfrak{g}$-quasi-Poisson manifold $\left(M, \pi_{r}, Z\right)$ is symplectic, i.e. if $\pi_{r}^{\#}: T^{*} M \rightarrow T M$ is invertible and $Z=0$.

By a dynamical twist quantization of $r(\lambda)$ we mean a h-equivariant map $J=1 \otimes 1+O(\hbar)$ : $U \rightarrow \otimes^{2} U \mathfrak{g}[[\hbar]]$ satisfying the semi-classical limit condition

$$
J(\lambda)-J^{2,1}(\lambda)=\hbar r(\lambda)+O\left(\hbar^{2}\right) \quad(\lambda \in U)
$$

and the modified dynamical twist equation

$$
J^{12,3}(\lambda) *_{P B W} J^{1,2}\left(\lambda+\hbar h^{(3)}\right)=\Phi^{-1} J^{1,23}(\lambda) *_{P B W} J^{2,3}(\lambda) \quad(\lambda \in U) .
$$

where $\Phi \in\left(\otimes^{3} U \mathfrak{g}\right)^{\mathfrak{g}}[[\hbar]]$ is an associator quantizing $Z$, of which we know the existence from [5, Proposition 3.10]. Recall that $*_{P B W}$ is the Poincaré-Birkhoff-Witt star-product given on polynomial functions as the pull-back of the usual product in $U\left(\mathfrak{h}_{\hbar}\right)^{1}$ by the symmetrization map sym: $S(\mathfrak{h})[[\hbar]] \rightarrow U\left(\mathfrak{h}_{\hbar}\right)$. We also made use of the following notations:

$$
\begin{gathered}
J^{12,3}(\lambda):=(\Delta \otimes \mathrm{id})(J(\lambda)) \text { and } \\
J^{1,2}\left(\lambda+\hbar h^{(3)}\right):=\sum_{k \geq 0} \frac{\hbar^{k}}{k !} \sum_{i_{1} \cdots i_{k}} \frac{\partial^{k} J}{\partial \lambda^{i_{1}} \cdots \partial \lambda^{i_{k}}}(\lambda) \otimes h_{i_{1}} \cdots h_{i_{k}} .
\end{gathered}
$$

In this paper we prove the following generalization of [13, Theorem 5.3] to the case of a nonabelian base:

\footnotetext{
${ }^{1} \mathfrak{h}_{\hbar}=\mathfrak{h}[[\hbar]]$ with bracket $[,]_{\hbar}:=\hbar[,]_{\mathfrak{h}}$.
} 
Theorem 0.1. Any symplectic dynamical $r$-matrix admits a dynamical twist quantization (with $\Phi=1)$.

Furthermore, two dynamical twist quantizations $J_{1}, J_{2}: U \rightarrow \otimes^{2} U \mathfrak{g}[[\hbar]]$ of $r$ are said to be gauge equivalent if there exists a $\mathfrak{h}$-equivariant map $T=1+O(\hbar): U \rightarrow U \mathfrak{g}[[\hbar]]$ such that

$$
T^{12}(\lambda) *_{P B W} J_{1}(\lambda)=J_{2}(\lambda) *_{P B W} T^{1}\left(\lambda+\hbar h^{(2)}\right) *_{P B W} T^{2}(\lambda) .
$$

In this context we also prove the following generalization of [13, Section 6] to the case of a nonabelian base, asserting that dynamical twist quantizations are classified by the second dynamical r-matrix cohomology (see Definition 3.2):

Theorem 0.2. Let $r$ be a symplectic dynamical $r$-matrix. Then the space of gauge equivalence classes of dynamical twist quantizations of $r$ (with $\Phi=1)$ is an affine space modeled on $H_{r}^{2}(U, \mathfrak{g})[[\hbar]]$.

A class of examples of symplectic dynamical $r$-matrices is given by nondegenerate reductive splittings. A reductive splitting $\mathfrak{g}=\mathfrak{h} \oplus \mathfrak{m}$ (with $[\mathfrak{h}, \mathfrak{m}] \subset \mathfrak{m}$ ) is called nondegenerate if there exists $\lambda \in \mathfrak{h}^{*}$ for which $\omega(\lambda) \in \wedge^{2} \mathfrak{m}^{*}$ defined by $\omega(\lambda)(x, y)=<\lambda,[x, y]_{\mid \mathfrak{h}}>$ is nondegenerate. Then $r_{\mathfrak{h}}^{\mathfrak{m}}(\lambda):=-\omega(\lambda)^{-1} \in \wedge^{2} \mathfrak{m} \subset \wedge^{2} \mathfrak{g}$ defines a symplectic dynamical $r$-matrix on the invariant open subset $\{\lambda \mid \operatorname{det} \omega(\lambda) \neq 0\} \subset \mathfrak{h}^{*}$ (see [11, Proposition 1] or [14, Theorem 2.3]; see also [7, Proposition 1.1] for a more algebraic proof). Moreover, one can use it to "compose" dynamical $r$-matrices (see [11, Proposition 1] and [7, Proposition 0.1]):

Proposition 0.3 (The composition formula). Assume that $\mathfrak{h}=\mathfrak{t} \oplus \mathfrak{m}$ is a nondegenerate reductive splitting and let $r_{\mathfrak{t}}^{\mathfrak{m}}: \mathfrak{t}^{*} \supset V \rightarrow \wedge^{2} \mathfrak{h}$ be the corresponding symplectic dynamical $r$-matrix. If $\rho: \mathfrak{h}^{*} \supset U \rightarrow \wedge^{2} \mathfrak{g}$ is a dynamical $r$-matrix with $Z \in\left(\wedge^{3} \mathfrak{g}\right)^{\mathfrak{g}}$, then

$$
\theta_{\rho}:=r_{\mathfrak{t}}^{\mathfrak{m}}+\rho_{\mid \mathfrak{t}^{*}}: \mathfrak{t}^{*} \supset U \cap V \longrightarrow \wedge^{2} \mathfrak{g}
$$

is a dynamical $r$-matrix with the same $Z$.

We prove that one can "quantize" the map $\rho \mapsto \theta_{\rho}$ :

Theorem 0.4. With the hypothesis of Proposition 0.3, there exists a map

$$
\Theta:\{\text { Dynamical twist quantizations of } \rho\} \longrightarrow\left\{\text { Dynamical twist quantizations of } \theta_{\rho}\right\}
$$

which keeps the associator $\Phi$ fixed.

The paper is organized as follows.

In Section 1 we first recall basic facts about quasi-Poisson manifolds, compatible quantizations and related results. We then give a sufficient condition for the existence of dynamical twist quantizations.

In Section 2 we give a very short proof of Theorem 0.1, using a result stating the existence of a quantum momentum map (see also [10, 12]) which is based on Fedosov's well-known globalization procedure [8, 9]. We start the section with a summary of basic ingredients of Fedosov's construction.

In Section 3 we prove Theorem 0.2 using a variant of the well-established classification of star-products on a symplectic manifold by formal series with coefficients in the second De Rham cohomology group of the manifold.

In Section 4 we prove a quantum analogue of the composition formula for classical dynamical $r$-matrices. We start with a new proof of the classical composition formula (Proposition 0.3) using (quasi-)Poisson reduction. We then derive its quantum counterpart (Theorem 0.4) using quantum reduction.

Notations. We denote by $\mathcal{O}_{\mathfrak{h}^{*}}=S(\mathfrak{h})$ polynomial functions on $\mathfrak{h}^{*}, \mathcal{O}_{U}=C^{\infty}(U) \supset \mathcal{O}_{\mathfrak{h}^{*}}$ smooth functions on $U \subset \mathfrak{h}^{*}$, and $\mathcal{O}_{G}$ the ring of smooth functions on the (eventually formal) Lie group $G$. For any element $x \in \mathfrak{g}$ we denote by $\vec{x}$ (resp. $\overleftarrow{x}$ ) the corresponding left (resp. 
right) invariant vector field on $G$.

Acknowledgements. We thank Benjamin Enriquez for pointing to us the problem of the quantum composition formula and for a very stimulating series of discussions during his short visit in Geneva. A.A. acknowledges the support of the Swiss National Science Foundation. D.C. thanks the Section de Mathématiques de l'Université de Genève, the Institut des Hautes Études Scientifiques and the Max-Planck-Institut für Mathematik Bonn where parts of this work were done.

\section{A SUfFicient CONDition For the Existence of a Dynamical tWist QUANTIZATION}

Let $r: \mathfrak{h}^{*} \supset U \rightarrow \wedge^{2} \mathfrak{g}$ be a coboundary dynamical $r$-matrix. Denote by $\pi_{r}$ the bivector field on $M=U \times G$ given by (0.1).

1.1. Quasi-Poisson manifolds and their quantizations. Recall from 1, 2, that a (g-) quasi-Poisson manifold is a manifold $X$ together with a $\mathfrak{g}$-action $\rho: \mathfrak{g} \rightarrow \mathfrak{X}(X)$, an invariant bivector field $\pi \in \Gamma\left(X, \wedge^{2} T X\right)^{\mathfrak{g}}$ and an element $Z \in\left(\wedge^{3} \mathfrak{g}\right)^{\mathfrak{g}}$ such that

$$
[\pi, \pi]=\rho(Z) .
$$

Let $\{f, g\}:=<\pi, d f \wedge d g>\left(f, g \in \mathcal{O}_{X}\right)$ be the corresponding quasi-Poisson bracket. Then equation (1.1) is equivalent to

$$
\{\{f, g\}, h\}+\{\{h, f\}, g\}+\{\{g, h\}, f\}=<\rho(Z), d f \wedge d g \wedge d h>\quad\left(f, g, h \in \mathcal{O}_{X}\right) .
$$

One can define the quasi-Poisson cochain complex of $(X, \pi, Z)$ as follows: $k$-cochains are $C_{\pi}^{k}(X)=\Gamma\left(X, \wedge^{k} T X\right)^{\mathfrak{g}}$ and the differential is $d_{\pi}=[\pi,-]$. The fact that $d_{\pi} \circ d_{\pi}=0$ follows from an easy calculation:

$$
d_{\pi} \circ d_{\pi}(x)=[\pi,[\pi, x]]=\frac{1}{2}[[\pi, \pi], x]=\frac{1}{2}[\rho(Z), x]=0 \quad\left(x \in C_{\pi}^{k}(X)\right) .
$$

Let us now fix an associator $\Phi \in\left(\otimes^{3} U \mathfrak{g}\right)^{\mathfrak{g}}[[\hbar]]$ quantizing $Z$ (we know it exists from [5, Proposition 3.10]). Following [6, Definition 4.4], by a quantization of a given quasi-Poisson manifold $(X, \pi, Z)$ we mean a series $* \in \operatorname{Bidiff}(X)^{\mathfrak{g}}[[\hbar]]$ of invariant bidifferential operators such that

- $f * g=f g+O(\hbar)$ for any $f, g \in \mathcal{O}_{X}$,

- $f * g-g * f=\hbar\{f, g\}+O\left(\hbar^{2}\right)$ for any $f, g \in \mathcal{O}_{X}$, and

- if we write $m_{*}(f \otimes g):=f * g$ for $f, g \in \mathcal{O}_{X}$, and $\widetilde{\Phi}:=S^{\otimes 3}\left(\Phi^{-1}\right)$, then 2

$$
m_{*} \circ\left(m_{*} \otimes \mathrm{id}\right)=m_{*} \circ\left(\mathrm{id} \otimes m_{*}\right) \circ \rho^{\otimes 3}(\widetilde{\Phi}) .
$$

Here, $S$ denotes the antipode of $U \mathfrak{g}$.

One has a natural notion of gauge transformation for quantizations. It is given by an element $Q=\mathrm{id}+O(\hbar) \in \operatorname{Diff}(X)^{\mathfrak{g}}[[\hbar]]$ that act on $*$ 's in the usual way:

$$
f *^{(Q)} g:=Q^{-1}(Q(f) * Q(g)) \quad\left(f, g \in \mathcal{O}_{X}\right) .
$$

More precisely, if $(\Phi, *)$ is a quantization of $(Z, \pi)$ then $\left(\Phi, *^{(Q)}\right)$ is also. In this case we say that $*$ and $*^{(Q)}$ are gauge equivalent.

\footnotetext{
${ }^{2}$ We thank Pavel Etingof for pointing to us that one has to use $\widetilde{\Phi}$ instead of $\Phi$ in this definition.
} 
1.2. Classical and quantum momentum maps. Let $(X, \pi, Z)$ be g-quasi-Poisson manifold and let $\mathcal{G}$ be a Lie algebra with Lie group $\mathbf{G}$. A momentum map is a smooth $\mathfrak{g}$-invariant map $\mu: M \rightarrow \mathcal{G}^{*}$ such that $\mu_{*} \pi=\pi_{\text {lin }}$ and for which the corresponding infinitesimal action $\mathcal{G} \rightarrow \mathfrak{X}(X) ; x \mapsto\left\{\mu^{*} x,-\right\}$ integrates to a right action of $\mathbf{G}$.

Let us describe the reduction procedure with respect to a given momentum map $\mu$. First of all $\mathbf{G}$ acts on $\mu^{-1}(0)$ and hence one can define the reduced space $X_{\text {red }}:=\mu^{-1}(0) / \mathbf{G}$. Let us assume that it is smooth (this is the case when 0 is a regular value and $\mathbf{G}$ acts freely); its function algebra is $\mathcal{O}_{X_{\text {red }}}=\mathcal{O}_{X}^{\mathcal{G}} /\left(\mathcal{O}_{X}^{\mathcal{G}} \cap \mathcal{I}_{0}\right)$, where $\mathcal{I}_{0}$ is the ideal generated by $\operatorname{im}\left(\mu^{*}\right)$. Since $\mu$ is $\mathfrak{g}$-invariant then $\mathfrak{g}$ acts on $\mu^{-1}(0)$. Moreover the $\mathfrak{g}$-action and the $\mathcal{G}$-action commute (because $\pi$ and $\mu$ are $\mathfrak{g}$-invariant), consequently $\mathfrak{g}$ also acts on $X_{\text {red }}$. Now observe that $\mathcal{O}_{X}^{\mathcal{G}}=\left\{f \in \mathcal{O}_{X} \mid\left\{f, \mathcal{I}_{0}\right\} \subset \mathcal{I}_{0}\right\}$, therefore the quasi-Poisson bracket $\{$,$\} naturally induces a$ quasi-Poisson bracket (with the same $Z$ ) on $\mathcal{O}_{X_{\text {red }}}$. In other words, $X_{\text {red }}$ inherits a structure of a quasi-Poisson manifold from the one of $X$.

Now assume that we are given a quantization $(*, \Phi)$ of the quasi-Poisson manifold $(X, \pi, Z)$. By a quantum momentum map quantizing $\mu$ we mean a map of algebras

$$
\mathbf{M}=\mu^{*}+O(\hbar):\left(\mathcal{O}_{\mathcal{G}^{*}}[[\hbar]], *_{P B W}\right) \longrightarrow\left(\mathcal{O}_{X}[[\hbar]], *\right)
$$

taking its values in $\mathfrak{g}$-invariant functions, and such that for any $f \in \mathcal{O}_{X}$ and any $x \in \mathcal{G}$ one has $[\mathbf{M}(x), f]_{*}=\hbar\left\{\mu^{*} x, f\right\}$.

Remark 1.1. One only needs to know $\mathbf{M}$ on linear functions $x \in \mathcal{G}$.

Let us describe the quantum reduction with respect to a given quantum momentum map M. First denote by $\mathcal{I}$ the right ideal generated by $\operatorname{im}(\mathbf{M})$ in $\left(\mathcal{O}_{X}[[\hbar]], *\right)$ and observe that its normalizer is $\mathcal{O}_{X}^{\mathfrak{h}}[[\hbar]]$. Therefore $\mathcal{O}_{X}^{\mathfrak{h}}[[\hbar]] \cap \mathcal{I}$ is a two-sided ideal in $\mathcal{O}_{X}^{\mathfrak{h}}[[\hbar]]$, and we can define the reduced algebra $\mathcal{A}:=\mathcal{O}_{X}^{\mathfrak{h}}[[\hbar]] / \mathcal{O}_{X}^{\mathfrak{h}}[[\hbar]] \cap \mathcal{I}$. Since $\mathcal{I} \cong \mathcal{I}_{0}[[\hbar]]$ then $\mathcal{A} \cong \mathcal{O}_{X_{\text {red }}}[[\hbar]]$ (as $\mathbb{R}[[\hbar]]$-modules). It is easy to see that the induced product on $\mathcal{O}_{X_{\text {red }}}[[\hbar]]$, together with $\Phi$, gives a quantization of the quasi-Poisson structure on $X_{\text {red }}$.

A quantization $*$ of a quasi-Poisson manifold $(M, \pi, Z)$ with a momentum map $\mu: M \rightarrow$ $\mathcal{G}^{*}$ for which $\mu^{*}$ itself defines a quantum momentum map (it will be $\mathbf{M}=U\left(\mu^{*}\right) \circ$ sym) is called strongly (G-)invariant.

1.3. Compatible quantizations. Let us first observe that $\pi_{r}$ defines a quasi-Poisson structure on $M=U \times G$. Here the action is $\mathfrak{g} \ni x \mapsto \overleftarrow{x} \in \mathfrak{X}(M)$ (it generates left translations). Remark that since $Z \in\left(\wedge^{3} \mathfrak{g}\right)^{\mathfrak{g}}$ then $\overleftarrow{Z}=\vec{Z}$. Moreover, the natural map $M \rightarrow \mathfrak{h}^{*},(\lambda, g) \mapsto \lambda$ is a momentum map and the corresponding right $H$-action is given by $(\lambda, g) \cdot h:=\left(\operatorname{Ad}_{h}^{*} \lambda, g h\right)$ (following the notation of the previous $\S$ we have $\mathcal{G}=\mathfrak{h}$ ). Conversely,

Proposition 1.2 ([14], Proposition 2.1). A map $\rho \in C^{\infty}\left(U, \wedge^{2} \mathfrak{g}\right)$ is a coboundary classical dynamical r-matrix if and only if

$$
\pi=\pi_{l i n}+\sum \frac{\partial}{\partial \lambda^{i}} \wedge \overrightarrow{h_{i}}+\overrightarrow{\rho(\lambda)}
$$

defines a $\mathfrak{g}$-quasi-Poisson structure on $U \times G$.

Proof. The proof given in [14] is for the case when $Z=0$, but it admits a straightforward generalization.

Following Ping Xu ([14]), by a compatible quantization of $\pi_{r}$ we mean a quantization $*^{\prime}$ which is such that for any $u, v \in \mathcal{O}_{\mathfrak{h}^{*}}$ and any $f \in \mathcal{O}_{G}, u *^{\prime} v=u * P B W v, f *^{\prime} u=f u$ and

$$
u *^{\prime} f=\sum_{k \geq 0} \frac{\hbar^{k}}{k !} \sum_{i_{1}, \ldots, i_{k}} \frac{\partial^{k} u}{\partial \lambda^{i_{1}} \cdots \partial \lambda^{i_{k}}} \overrightarrow{h_{i_{1}}} \cdots \overrightarrow{h_{i_{k}}} \cdot f .
$$


Proposition 1.3. There is a bijective correspondence between compatible quantizations of $\pi_{r}$ and dynamical twist quantizations of $r$.

Proof. Let $*^{\prime}$ be a compatible quantization of $\pi_{r}$. Since $*^{\prime}$ is $G$-invariant then for all $f, g \in \mathcal{O}_{G}$ one has

with $J: U \rightarrow \otimes^{2} U \mathfrak{g}[[\hbar]]$. Moreover

$$
\left(f *^{\prime} g\right)(\lambda)=\overrightarrow{J(\lambda)}(f, g) \quad\left(\lambda \in \mathfrak{h}^{*}\right)
$$

Lemma 1.4. $*^{\prime}$ is strongly $\mathfrak{h}$-invarian 3 .

Proof of the lemma. Let $f=g u\left(g \in \mathcal{O}_{G}\right.$ and $\left.u \in \mathcal{O}_{\mathfrak{h}^{*}}\right)$ on $U \times G$. Then for any $h \in \mathfrak{h}$ one has

$$
\begin{aligned}
h *^{\prime} f-f *^{\prime} h & =h *^{\prime}(g u)-(g u) *^{\prime} h=h *^{\prime}\left(g *^{\prime} u\right)-\left(g *^{\prime} u\right) *^{\prime} h \\
& =\left(h *^{\prime} g\right) *^{\prime} u-g *^{\prime}\left(u *^{\prime} h\right) \quad(\Phi \text { acts trivialy }) \\
& =\left(g *^{\prime} h+\hbar(\vec{h} \cdot g)\right) *^{\prime} u-g *^{\prime}\left(u *^{\prime} h\right) \\
& =g *^{\prime}\left([h, u]_{*^{\prime}}\right)+\hbar\left(\chi_{h} \cdot g\right) *^{\prime} u=g\left([h, u]_{*_{P B}}\right)+\hbar\left(\chi_{h} \cdot g\right) u \\
& =\hbar\left(g\left(\chi_{h} \cdot u\right)+\left(\chi_{h} \cdot g\right) u\right)=\hbar\left(\chi_{h} \cdot f\right)
\end{aligned}
$$

Hence for any $f \in \mathcal{O}_{M}, h * f-f * h=\hbar\left(\chi_{h} \cdot f\right)$.

Therefore using [14, Proposition 3.2] one obtains that $J$ is $H$-equivariant. The following lemma ends the first part of the proof:

Lemma 1.5. J satisfies the dynamical twist equation.

Proof of the lemma. Let us define $\mathbf{L}: \mathfrak{g} \ni x \mapsto \vec{x}$ and $\mathbf{R}: \mathfrak{g} \ni x \mapsto \overleftarrow{x}$, and denote by $m^{(n)}: \mathcal{O}_{M}^{\otimes n} \rightarrow \mathcal{O}_{M} ; f_{1} \otimes \cdots \otimes f_{n} \mapsto f_{1} \cdots f_{n}$ the standard $n$-fold product of functions. A computation in [14] emphases the fact that for all $f, g, h \in \mathcal{O}_{G}$, one has 4

$$
m_{*^{\prime}} \circ\left(m_{*^{\prime}} \otimes \mathrm{id}\right)(f \otimes g \otimes h)=\overrightarrow{J^{12,3}(\lambda) *_{P B W} J^{1,2}\left(\lambda+\hbar h^{(3)}\right)}(f \otimes g \otimes h)
$$

and

Therefore,

$$
m_{*^{\prime}} \circ\left(\mathrm{id} \otimes m_{*^{\prime}}\right)(f \otimes g \otimes h)=\overrightarrow{J^{1,23}(\lambda) *_{P B W} J^{2,3}(\lambda)}(f \otimes g \otimes h) .
$$

$$
\begin{aligned}
m_{*^{\prime}} \circ\left(\mathrm{id} \otimes m_{*^{\prime}}\right) \circ \mathbf{R}^{\otimes 3}(\widetilde{\Phi})(f \otimes g \otimes h) & =m^{(3)}\left(\mathbf{L}^{\otimes 3}\left(J^{1,23}(\lambda) *_{P B W} J^{2,3}(\lambda)\right) \mathbf{R}^{\otimes 3}(\widetilde{\Phi})(f \otimes g \otimes h)\right) \\
& =m^{(3)}\left(\mathbf{R}^{\otimes 3}(\widetilde{\Phi}) \mathbf{L}^{\otimes 3}\left(J^{1,23}(\lambda) *_{P B W} J^{2,3}(\lambda)\right)(f \otimes g \otimes h)\right) \\
& =\overleftrightarrow{S^{\otimes 3}\left(\Phi^{-1}\right)}\left(\mathbf{L}^{\otimes 3}\left(J^{1,23}(\lambda) *_{P B W} J^{2,3}(\lambda)\right)(f \otimes g \otimes h)\right) \\
& =\overrightarrow{\Phi^{-1}}\left(\mathbf{L}^{\otimes 3}\left(J^{1,23}(\lambda) *_{P B W} J^{2,3}(\lambda)\right)(f \otimes g \otimes h)\right) \\
& =\overrightarrow{\Phi^{-1} J^{1,23}(\lambda) *_{P B W} J^{2,3}(\lambda)}(f \otimes g \otimes h),
\end{aligned}
$$

where the equality before the last one follows from the invariance of $\Phi$. This ends the proof of the lemma.

Conversely, let $J=\sum_{\alpha} f_{\alpha} A_{\alpha} \otimes B_{\alpha}$ be a dynamical twist quantization of $r\left(f_{\alpha} \in \mathcal{O}_{U}[[\hbar]]\right.$ and $\left.A_{\alpha}, B_{\alpha} \in U \mathfrak{g}\right)$. Following [14] we define a $G$-invariant product $*^{\prime}$ on $\mathcal{O}_{M}[[\hbar]]$ by

$$
g_{1} *^{\prime} g_{2}:=\sum_{k \geq 0, \alpha} \frac{\hbar^{k}}{k !} \sum_{i_{1}, \ldots, i_{k}} f_{\alpha} *_{P B W}\left(\overrightarrow{A_{\alpha}} \cdot \frac{\partial^{k} g_{1}}{\partial \lambda^{i_{1}} \cdots \partial \lambda^{i_{k}}}\right) *_{P B W}\left(\overrightarrow{B_{\alpha}} \overrightarrow{h_{i_{1}}} \ldots \overrightarrow{h_{i_{k}}} \cdot g_{2}\right) \text {. }
$$

\footnotetext{
${ }^{3}$ In particular $*^{\prime}$ is $H$-invariant. It was not noticed in [14], where the definition of compatible star-products includes this $H$-invariance property. The lemma claims that it comes for free (like in the classical situation).

${ }^{4}$ The reader must pay attention to the following important remark: for any $P \in \otimes^{n} U \mathfrak{g}$ we denote by $\vec{P}$ (resp. $\overleftarrow{P}$ ) the corresponding left (resp. right) invariant multidifferential operator, while $\mathbf{L}^{\otimes n}(P)$ (resp. $\left.\mathbf{R}^{\otimes n}(P)\right)$ is an element in $\otimes^{n} \operatorname{Diff}(G)^{G_{\text {left }}}$ (resp. $\left.\otimes^{n} \operatorname{Diff}(G)^{G_{\text {right }}}\right)$. Namely, $\vec{P}=m^{(n)} \circ\left(\mathbf{L}{ }^{\otimes n}(P)\right)$.
} 
One can check by direct computations that $\mathfrak{h}$-equivariance of $J$ implies strong $\mathfrak{h}$-invariance of $*^{\prime}$, and that the dynamical twist equation implies equation (1.2).

Remark 1.6. Since $\mathcal{O}_{\mathfrak{h}^{*}}=S(\mathfrak{h})$ is generated as a vector space by $h^{n}, h \in \mathfrak{h}$ and $n \in \mathbb{N}$, then one can rewrite condition (1.3) as

$$
h^{n} *^{\prime} f=\sum_{k=0}^{n} \hbar^{k} C_{n}^{k}\left(\vec{h}^{k} \cdot f\right) h^{n-k} .
$$

We saw in Lemma 1.4 that a compatible quantization always satisfies the strongly $\mathfrak{h}$ invariance condition. In what follows we show that this condition is actually sufficient for the existence of a compatible quantization.

\subsection{A sufficient condition for the existence of a compatible quantization.}

Proposition 1.7. Assume that we are given a strongly $\mathfrak{h}$-invariant quantization $*$ of $\pi_{r}$ on $M$. Then there exists a gauge equivalent compatible quantization $*^{\prime}$ of $\pi_{r}$. Therefore there exists a dynamical twist quantization $J$ of $r$.

Proof. First observe that $h * h^{\prime}-h^{\prime} * h=\hbar\left[h, h^{\prime}\right]_{\mathfrak{h}}=\left[h, h^{\prime}\right]_{\mathfrak{h}_{\hbar}}$. Therefore we have an algebra morphism

$$
a: U\left(\mathfrak{h}_{\hbar}\right) \longrightarrow\left(\mathcal{O}_{M}, *\right) .
$$

Then define the algebra morphism $Q: \mathcal{O}_{\mathfrak{h}^{*} \times G}=S(\mathfrak{h}) \otimes \mathcal{O}_{G} \longrightarrow \mathcal{O}_{M}$ as follows:

$$
Q(f u)=f * a(\operatorname{sym}(u)) \quad\left(u \in S(\mathfrak{h}), f \in \mathcal{O}_{G}\right),
$$

where sym $: S(\mathfrak{h})[[\hbar]] \longrightarrow U\left(\mathfrak{h}_{\hbar}\right)$ is the isomorphism sending $h^{n}$ to $h^{n}$ for any $h \in \mathfrak{h}$. Thus $Q\left(h^{n} \otimes f\right)=f * \underbrace{h * \cdots * h}_{n \text { times }}$, and since $*$ can be expressed as a series $m_{0}+O(\hbar)$ of bidifferential operators on $M$ then $Q$ can be expressed as a series id $+O(\hbar)$ of differential operators on $M$. Moreover it is obviously $\mathfrak{g}$-invariant (since $*$ is), consequently we have a new quantization $*^{\prime}$ of $\pi_{r}$, gauge equivalent to $*$, defined as follows: for any $f, g \in \mathcal{O}_{M}$,

$$
f *^{\prime} g=Q^{-1}(Q(f) * Q(g)) .
$$

Let us now check that $*^{\prime}$ satisfies all Xu's properties for compatible quantizations.

- for any $u, v \in S(\mathfrak{h})$,

$$
\begin{aligned}
u *^{\prime} v & =Q^{-1}(a(\operatorname{sym}(u)) * a(\operatorname{sym}(v)) \\
& =Q^{-1}(a(\operatorname{sym}(u) \operatorname{sym}(v))) \\
& =Q^{-1}\left(a\left(\operatorname{sym}\left(u *_{P B W} v\right)\right)\right)=u *_{P B W} v
\end{aligned}
$$

- let $u \in S(\mathfrak{h})$ and $f \in \mathcal{O}_{G}$, then $f *^{\prime} u=Q^{-1}(f * a(\operatorname{sym}(u)))=f u$. Let us now compute $u *^{\prime} f$; we can assume that $u=h^{n}, h \in \mathfrak{h}$, and then

$$
\begin{aligned}
u *^{\prime} f & =Q^{-1}(a(\operatorname{sym}(u)) * f)=Q^{-1}(\underbrace{h * \cdots * h}_{n \text { times }} * f) \\
& =Q^{-1}(\sum_{k=0}^{n} C_{n}^{k} \hbar^{k}\left(\vec{h}^{k} \cdot f\right) * \underbrace{h * \cdots * h}_{n-k \text { times }}) \\
& =\sum_{k=0}^{n} \hbar^{k} C_{n}^{k}\left(\vec{h}^{k} \cdot f\right) h^{n-k}
\end{aligned}
$$

- $\operatorname{since} *$ is a $H$-invariant star-product, then $Q$ is a $H$-invariant gauge equivalence. Therefore $*^{\prime}$ is also $H$-invariant.

The proposition is proved.

Remark 1.8. The gauge transformation $Q$ constructed above obviously satisfies $Q(h)=h$ for any $h \in \mathfrak{h}$. 


\section{QuAntization of SyMPLECTIC DYNAMiCAL $r$-MATRiCES}

In this section we prove Theorem 0.1 . We start by recalling Fedosov's construction of star-products on a symplectic manifold (for more details we refer to [8, 9]).

2.1. Fedosov's star-products. Let $(M, \omega)$ a symplectic manifold and denote by $\pi=\omega^{-1}$ the corresponding Poisson bivector. Then its tangent bundle $T M$ inherits a Poisson structure $\tilde{\pi}$ expressed locally as

$$
\tilde{\pi}=\pi^{i j}(x) \frac{\partial}{\partial y^{i}} \wedge \frac{\partial}{\partial y^{j}},
$$

where $y^{i}$ s are coordinates in the fibers. This Poisson structure is regular and constant on the symplectic leaves which are the fibers $T_{x} M$ of the bundle. Therefore it is quantized by the series of fiberwize bidifferential operators $\exp (\hbar \tilde{\pi})$. It defines an associative product ○ on sections of $W=\hat{S}\left(T^{*} M\right)[[\hbar]]$ that naturally extends to $\Omega^{*}(M, W)$. The center of $\left(\Omega^{*}(M, W), \circ\right)$ consists of forms that are constant in the fibers, i.e. lying in $\Omega^{*}(M)[[\hbar]]$.

By assigning the degree $2 k+l$ to sections of $\hbar^{k} S^{m}\left(T^{*} M\right)$ there is a natural decreasing filtration

$$
W=W_{0} \supset W_{1} \supset \cdots \supset W_{i} \supset W_{i+1} \supset \cdots \supset \mathcal{O}_{M} \cdot
$$

Now fix (once and for all) a torsion free connection $\nabla$ on $M$ with Christoffel's symbols $\Gamma_{i j}^{k}$. One can assume without loss of generality that it is symplectic (see [9, Section 2.5]), which means that $\omega$ is parallel w.r.t. $\nabla$. Then consider

$$
\partial: \Omega^{*}(M, W) \rightarrow \Omega^{*+1}(M, W)
$$

its induced covariant derivative. In Darboux local coordinates we have

$$
\partial=d+\frac{1}{\hbar}[\Gamma,-]_{0}
$$

where $\Gamma=-\frac{1}{2} \Gamma_{i j k} y^{i} y^{j} d x^{k}$ is a local 1 -form with values in $W\left(\Gamma_{i j k}=\omega_{i l} \Gamma_{j k}^{l}\right)$. One has

$$
\partial^{2}=-\frac{1}{\hbar}[R,-] 。
$$

where $R=\frac{1}{4} R_{i j k l} y^{i} y^{j} d x^{k} \wedge d x^{l}$, and $R_{i j k l}=\omega_{i m} R_{j k l}^{m}$ is the curvature tensor of the symplectic connection $\nabla$.

Let us consider more general derivations of $\left(\Omega^{*}(M, W), \circ\right)$ of the form

$$
D=\partial-\delta+\frac{1}{\hbar}[r,-] 。
$$

where $r \in \Omega^{1}(M, W)$ and $\delta=\frac{1}{\hbar}\left[\omega_{i j} y^{i} d x^{j},-\right]_{\mathrm{o}}$. A simple calculation yields that

$$
D^{2}=-\frac{1}{\hbar}[\Omega,-] \text { 。 }
$$

where $\Omega=\omega+R+\delta r-\partial r-\frac{1}{\hbar} r^{2} \in \Omega^{2}(M, W)$ is called the Weyl curvature of $D$. In particular $D$ is flat (i.e. $D^{2}=0$ ) if and only if $\Omega \in \Omega^{2}(M)[[\hbar]]$ (i.e. is a central 2-form), and in this case the Bianchi identity for $\nabla$ implies that $d \Omega=D \Omega=0$.

In computing $D^{2}$ one sees that $\delta: \Omega^{*}\left(M, W_{k}\right) \rightarrow \Omega^{*+1}\left(M, W_{k-1}\right)$ has square zero and that the torsion freeness of $\nabla$ implies $\delta \partial+\partial \delta=0$. Then we define a homotopy operator $\kappa: \Omega^{*}\left(M, W_{k}\right) \rightarrow \Omega^{*-1}\left(M, W_{k+1}\right)$ on monomials $a \in \Omega^{p}\left(M, S^{q}\left(T^{*} M\right)\right)$ : if $p+q \neq 0$ then

$$
\kappa(a)=\frac{1}{p+q} y^{i} \partial_{d x^{i}} a
$$

and otherwise $\kappa(a)=0$. One easily check that $\kappa^{2}=0$ and $\delta \kappa+\kappa \delta=\mathrm{id}-\sigma$ where

$$
\sigma: \Omega^{*}(M, W) \rightarrow C^{\infty}(M)[[\hbar]], a \mapsto a_{\mid d x^{i}=y^{i}=0}
$$

is the projection onto 0 -forms constant in the fibers. 
Theorem 2.1 (Fedosov). For any closed 2 -form $\Omega=\omega+O(\hbar) \in Z^{2}(M)[[\hbar]]$ there exists a unique $r \in \Omega^{1}\left(M, W_{3}\right)$ such that $\kappa(r)=0$ and

$$
D=\partial-\delta+\frac{1}{\hbar}[r,-]_{0}
$$

has Weyl curvature $\Omega$ and is therefore flat.

Proof. First observe that $\Omega=\omega+R+\delta r-\partial r-\frac{1}{\hbar} r^{2}$ with $\kappa(r)=0$ if and only if

$$
r=\kappa\left(\Omega-\omega-R+\partial r+\frac{1}{\hbar} r^{2}\right) .
$$

Since $\partial$ preserves the filtration and $\kappa$ raises its degree by 1 then $\kappa(\Omega-\omega-R) \in \Omega^{1}\left(M, W_{3}\right)$ and the sequence $\left(r_{n}\right)_{n \geq 3}$ defined by the iteration formula

$$
r_{n+1}=r_{0}+\kappa\left(\partial r_{n}+\frac{1}{\hbar} r_{n}^{2}\right)
$$

with $r_{3}=\kappa(\Omega-\omega-R)$ converges to a unique element $r \in \Omega^{1}\left(M, W_{3}\right)$ which is a solution of equation (2.1). We have proved the existence.

Conversely, for any solution $r \in \Omega^{1}\left(M, W_{3}\right)$ of (2.1) define $r_{k}=r \bmod W_{k+1}$. Then $r_{3}=\kappa(\Omega-\omega-R)$ and the sequence $\left(r_{n}\right)_{n \geq 3}$ satisfies (2.2). Unicity is proved.

Such a flat derivation $D$ is called a Fedosov connection (of $\nabla$-type). The previous theorem claims that they are in bijection with series $\Omega$ of closed two forms starting with $\omega$.

Theorem 2.2 (Fedosov). If $D$ is a Fedosov connection then for any $f_{0} \in C^{\infty}(M)[[\hbar]]$ there exists a unique $D$-closed section $f \in \Gamma(M, W)$ such that $\sigma(f)=f_{0}$. Hence $\sigma$ establishes an isomorphism between $Z_{D}^{0}(W)$ and $C^{\infty}(M)[[\hbar]]$.

Proof. Let $f_{0} \in C^{\infty}(M)[[\hbar]]$. One has $D(f)=0$ with $\sigma(f)=f_{0}$ if and only if

$$
f=f_{0}+\kappa\left(\partial f+\frac{1}{\hbar}[r, f]_{\circ}\right) .
$$

Like in the proof of Theorem 2.1 we can solve (uniquely) this equation with the help of an iteration formula: $f_{n+1}=f_{0}+\kappa\left(\partial f_{n}+\frac{1}{\hbar}\left[r, f_{n}\right]_{0}\right)$.

Then $f * g=\sigma\left(\sigma^{-1}(f) \circ \sigma^{-1}(g)\right)$ defines a star-product on $C^{\infty}(M)[[\hbar]]$ that quantizes $(M, \omega)$. A star-product constructed this way is called a Fedosov star-product (of $\nabla$-type) and is uniquely determined, once $\nabla$ is fixed, by its characteristic 2 -form

$$
\omega_{\hbar}:=\frac{1}{\hbar}(\Omega-\omega) \in Z^{2}(M)[[\hbar]] .
$$

Moreover one can easily prove the following

Lemma $2.3([4])$. Let $\omega_{\hbar}^{(i)}=\sum_{k>0} \hbar^{k-1} \omega_{k}^{(i)} \in Z^{2}(M)[[\hbar]](i=1,2)$ and denote by $*_{i}$ the Fedosov star-product with characteristic two-form $\omega_{\hbar}^{(i)}$. If $\omega_{\hbar}^{(1)}=\omega_{\hbar}^{(2)}+O\left(\hbar^{k}\right)$ then

$$
*^{(1)}-*^{(2)}=\hbar^{k+1} \pi^{\#}\left(\omega_{k}^{(1)}-\omega_{k}^{(2)}\right)+O\left(\hbar^{k+2}\right) .
$$

2.2. Fedosov's construction in the presence of symmetries. Let $(M, \omega)$ a symplectic manifold. Let us prove two results on the compatibility of Fedosov's construction with group actions and hamiltonian vector fields.

Proposition 2.4 (Fedosov). Assume that a group $G$ acts on $(M, \omega)$ by symplectomorphisms and is equipped with a $G$-invariant torsion free connection. Then for any $\omega_{\hbar} \in Z^{2}(M)^{G}[[\hbar]]$ the corresponding Fedosov star-product is G-invariant. 
Proof. First observe that starting from a $G$-invariant torsion free connection $\nabla$ one can assume without loss of generality that it is symplectic (see the proof of Proposition 5.2.2 in [9], where all expressions become obviously $G$-invariant).

Then, being a symplectomorphism of $(M, \omega)$, any element $g \in G$ acts via its differential $d g$ on $(T M, \tilde{\pi})$ as a Poisson automorphism linear in the fibers. Then its dual map $g^{*}: T^{*} M \rightarrow$ $T^{*} M$ defined by $<g^{*} \xi, x>=<\xi, d g(x)>$ extends to $W$ as an automorphism.

Finally, we need to prove that $g^{*}$ preserves the Fedosov connection with Weyl curvature $\Omega=\omega+\omega_{\hbar}$. On one hand the automorphism $g^{*}$ commutes with $\partial$ (since $\nabla$ is assumed to be $G$-invariant) and so $g^{*} R=R$. On the other hand $g^{*}$ also commutes with $\delta$ and $\kappa$, thus if $r$ is a solution of equation (2.1) with $\kappa(r)=0$ then so is $g^{*} r$. By uniqueness $g^{*} r=r$. We are done.

Proposition 2.5 (Fedosov). Let $H \in \mathcal{O}_{M}$ such that $\chi=\{H, \cdot\}$ preserves a torsion free connection on $M$. Then for any $\omega_{\hbar} \in Z^{2}(M)[[\hbar]]$ such that $\iota_{\chi} \omega_{\hbar}=0$ the corresponding Fedosov star-product $*$ satisfies $H * f-f * H=\hbar(\chi \cdot f)$ for any $f \in \mathcal{O}_{M}$.

Proof. First observe that $L_{\chi} \omega_{\hbar}=\left(d \iota_{\chi}+\iota_{\chi} d\right) \omega=0$. Therefore, the infinitesimal version of the previous proof ensures us that the Fedosov connection $D$ with Weyl curvature $\Omega=\omega+\omega_{\hbar}$ is $L_{X}$-equivariant. Hence in local Darboux coordinates it writes $D=d+\frac{1}{\hbar}[\gamma,-]$ 。 with $L_{\chi} \gamma=0$, and $\Omega=-d \gamma-\frac{1}{\hbar} \gamma^{2}$. Let us compute

$$
\begin{aligned}
D\left(H-\iota_{\chi} \gamma\right) & =d H-d \iota_{\chi} \gamma-\frac{1}{\hbar}\left[\gamma, \iota_{\chi} \gamma\right]_{\circ}=\iota_{\chi} \Omega+\iota_{\chi} d \gamma+\frac{1}{\hbar}\left[\iota_{\chi} \gamma, \gamma\right]_{\circ} \\
& =\iota_{\chi}\left(\Omega+d \gamma+\frac{1}{\hbar} \gamma^{2}\right)=0
\end{aligned}
$$

Since $\sigma\left(H-\iota_{\chi} \gamma\right)=\sigma(H)=H$, it means that $\sigma^{-1}(H)=H-\iota_{\chi} \gamma$ in local Darboux coordinates. Consequently, for any $f \in \mathcal{O}_{M}[[\hbar]]$

$$
\begin{aligned}
H * f-f * H & =\sigma\left(\left[H-\iota_{\chi} \gamma, \sigma^{-1}(f)\right]_{\circ}\right)=\sigma\left(-\left[\iota_{\chi} \gamma, \sigma^{-1}(f)\right]_{\circ}\right) \\
& =\sigma\left(-\iota_{\chi} \hbar(D-d) \sigma^{-1}(f)\right)=\hbar \sigma\left(\iota_{\chi} d \sigma^{-1}(f)\right) \\
& =\hbar \sigma\left(L_{\chi} \sigma^{-1}(f)-\iota_{\chi} \sigma^{-1}(f)\right)=\hbar L_{\chi}(f)=\hbar(\chi \cdot f)
\end{aligned}
$$

The proposition is proved.

2.3. Proof of Theorem 0.1. Let $r: U \rightarrow \wedge^{2} \mathfrak{g}$ a symplectic dynamical $r$-matrix. A basis $\mathcal{B}$ of vector fields on $M=U \times G$ is given by $\mathcal{B}=\left(\ldots, \partial_{\lambda^{i}}, \ldots, \ldots, \overrightarrow{e_{i}}, \ldots\right)$ where $\left(\lambda^{i}\right)_{i}$ is a base of $\mathfrak{h}^{*}$ and $\left(e_{i}\right)_{i}$ is a base of $\mathfrak{g}$. Then one defines a torsion free connection $\nabla$ on $M$ as

$$
\nabla_{b} X=\frac{1}{2}[b, X] \quad(b \in \mathcal{B}, X \in \mathfrak{X}(M)) .
$$

Remark that $\left[\chi_{h}, b\right] \in \operatorname{span}_{\mathbb{R}} \mathcal{B}$ for any $b \in \mathcal{B}$. Therefore it follows immediately from the Jacobi identity that $\nabla$ is $\mathfrak{h}$-invariant: for all $X, Y \in \mathfrak{X}(M)$ and $h \in \mathfrak{h}$,

$$
\left[\chi_{h}, \nabla_{X} Y\right]=\nabla_{\left[\chi_{h}, X\right]} Y+\nabla_{X}\left[\chi_{h}, Y\right] .
$$

Thus from Proposition 2.5 the Fedosov star-product $*$ with the trivial characteristic 2 -form is strongly $\mathfrak{h}$-invariant. Moreover $\nabla$ is obviously $G$-invariant, hence Proposition 2.4 implies that $*$ is also $G$-invariant.

Finally, we apply Proposition 1.7 to construct a compatible quantization of $\pi_{r}$. We are done.

\section{Classification}

Let $r: \mathfrak{h}^{*} \supset U \rightarrow \wedge^{2} \mathfrak{g}$ a dynamical $r$-matrix. Denote by $\pi_{r}$ the corresponding $H$-invariant $\mathfrak{g}$-quasi-Poisson structure (0.1) on $M=U \times G$ (together with $\left.Z \in\left(\wedge^{3} \mathfrak{g}\right)^{\mathfrak{g}}\right)$. 
3.1. Strongly invariant equivalences and obstructions. By a strongly invariant equivalence between two strongly $\mathfrak{h}$-invariant quantizations of $\pi_{r}$ we mean a $H$-invariant equivalence $Q$ (namely, $\left.Q=\mathrm{id}+O(\hbar) \in \operatorname{Diff}(\mathrm{M})^{G \times H}[[\hbar]]\right)$ satisfying $Q(h)=h$ for any $h \in \mathfrak{h} \subset \mathcal{O}_{M}$. We will now develop an analogue of the usual obstruction theory in this context.

Let us denote by $b$ the Hochschild coboundary operator for cochains on the (commutative) algebra $\mathcal{O}_{M}$. We start with the following result which is a variant of a standard one.

Proposition 3.1. Suppose that $*_{1}$ and $*_{2}$ are two strongly invariant quantizations of $\pi_{r}$ :

$$
f *_{i} g=\sum_{k \geq 0} \hbar^{k} C_{k}^{i}(f, g) \quad(i=1,2) .
$$

Assume that $*_{1}$ and $*_{2}$ coincide up to order $n$, i.e. $C_{k}^{1}=C_{k}^{2}$ if $k \leq n$. Then

(1) there exists $B \in \Gamma\left(M, \wedge^{2} T M\right)^{G \times H}$ and $E \in \operatorname{Diff}(M)^{G \times H}$ such that $B(h,-)=0$ and $E(h)=0$ if $h \in \mathfrak{h} \subset \mathcal{O}_{\mathfrak{h}^{*}},\left[\pi_{r}, B\right]=0$, and satisfying

$$
\left(C_{n+1}^{1}-C_{n+1}^{2}\right)(f, g)=B(f, g)+(b E)(f, g) \quad\left(f, g \in \mathcal{O}_{M}\right) ;
$$

(2) there exists $P \in \operatorname{Diff}(M)^{G \times H}$ such that $C_{1}=\pi_{r}+b P$ and $P(h)=0$ for $h \in \mathfrak{h}$;

(3) if $B=\left[\pi_{r}, X\right], X \in \mathfrak{X}(M)^{G \times H}$ such that $X(h)=0$, then the strongly invariant equivalence $Q=1+\hbar^{n} X+\hbar^{n+1}(E+[X, P])$ transforms $*_{1}$ into another strongly invariant star-product which coincides with $*_{2}$ up to order $n+1$.

Proof. We use a similar argument as in [13, 3, 4].

(1) It is well-known that $b\left(C_{n+1}^{1}-C_{n+1}^{2}\right)=0$. Hence we may write

$$
C_{n+1}^{1}-C_{n+1}^{2}=B+b\left(E_{0}\right)
$$

where $B \in \Gamma\left(M, \wedge^{2} T M\right)^{G \times H}$ is the skew-symmetric part of $C_{n+1}^{1}-C_{n+1}^{2}$ and $E_{0} \in \operatorname{Diff}(M)$. Moreover, one knows (see e.g. [4]) that $\left[\pi_{r}, B\right]=0$, and it follows directly from the strong $\mathfrak{h}$-invariance property for $*_{1}$ and $*_{2}$ that $B(h,-)=0$ if $h \in \mathfrak{h}$.

Since $U \times G$ admits a $G \times H$-invariant connection and $b\left(E_{0}\right)$ is obviously $G \times H$-invariant, then according to [3, Proposition 2.1] we can assume that $E_{0}$ is $G \times H$-invariant. In particular $E_{0}$ is $G$-invariant and hence $E_{0}(f), f \in \mathcal{O}_{U}$, is a function on $U$ only. Thus we can define a $H$-invariant vector field $\vec{v}$ on $U$ as follows: $\langle d h, \vec{v}\rangle=E_{0}(h)$ for any $h \in \mathfrak{h} \subset \mathcal{O}_{U}$. Now $E:=E_{0}-\vec{v}$ satisfies all the required properties and $b(E)=b\left(E_{0}\right)-b(\vec{v})=b\left(E_{0}\right)$.

(2) It is standard that $C_{1}=\pi_{r}+b\left(P_{0}\right)$. By repeating a similar argument as in (1) we can prove that $P_{0}$ can be chosen so that $P_{0}=P \in \operatorname{Diff}(M)^{G \times H}$ and satisfies $P(h)=0$ for any $h \in \mathfrak{h}$.

The third statement (3) follows from an easy (and standard) calculation.

This proposition means that obstructions to strongly invariant equivalences are in the second cohomology group of the subcomplex $C^{\infty}\left(U, \wedge^{*} \mathfrak{g}\right)^{\mathfrak{h}}$ in the $H$-invariant quasi-Poisson cochain complex of $\left(M, \pi_{r}, Z\right)$. On such cochains $c$ the (quasi-)Poisson coboundary operator $\left[\pi_{r},-\right]$ reduces to $d_{r}(c):=h_{i} \wedge \frac{\partial c}{\partial \lambda^{i}}+[r, c]$.

Definition 3.2. The cohomology $H_{r}^{*}(U, \mathfrak{g})$ of this cochain complex is called the dynamical $r$-matrix cohomology associated to $r: U \rightarrow \wedge^{2} \mathfrak{g}$.

3.2. Classification of strongly invariant star-products. Now assume that the quasiPoisson manifold $\left(M, \pi_{r}\right)$ is actually symplectic and denote by $\omega_{r}$ the symplectic form; it is $G \times H$-invariant and satisfies $\iota_{\chi_{h}} \omega=0$ for any $h \in \mathfrak{h} \subset \mathcal{O}_{\mathfrak{h}^{*}}$. The $G \times H$-invariant isomorphism

$$
\pi_{r}^{\#}: T^{*} M \stackrel{\sim}{\longrightarrow} T M
$$

extends to a $G \times H$-invariant isomorphism of cochain complexes

$$
\left(\Omega^{*}(M), d\right) \stackrel{\sim}{\longrightarrow}\left(\Gamma\left(M, \wedge^{*} T M\right),\left[\pi_{r},-\right]\right)
$$


which restricts to an isomorphism

$$
\left(\Omega_{\mathfrak{h}}^{*}(M)^{G}, d\right) \stackrel{\sim}{\longrightarrow}\left(C^{\infty}\left(U, \wedge^{*} \mathfrak{g}\right)^{\mathfrak{h}}, d_{r}\right),
$$

where $\Omega_{\mathfrak{h}}^{*}(M):=\left\{\alpha \in \Omega^{*}(M)^{H} \mid \iota_{\chi_{h}} \alpha=0, \forall h \in \mathfrak{h}\right\}$.

Let us fix once and for all a symplectic $G \times H$-invariant connection $\nabla$ on $M$ (we know it exists) and remember from the previous section that for any $\omega_{\hbar} \in \hbar \Omega^{2}(M)^{G \times H}[[\hbar]]$ such that $d \omega_{\hbar}=0$ there exists a (unique) $G \times H$-invariant Fedosov star-product $*$ with characteristic 2-form $\omega_{\hbar}$. Moreover, if $\omega_{\hbar} \in \Omega_{\mathfrak{h}}^{2}(M)[[\hbar]]$ then Proposition [2.5 implies that $*$ is strongly $\mathfrak{h}$-invariant. Therefore we can associate a strongly invariant quantization of $\pi_{r}$ (which is actually a Fedosov star-product) to any closed two form $\omega_{\hbar} \in \Omega_{\mathfrak{h}}^{2}(M)^{G}[[\hbar]]$.

In the rest of the section, all Fedosov star-products are assumed to be of $\nabla$-type and $G$-invariant (since they quantize the $\mathfrak{g}$-quasi-Poisson structure $\pi_{r}$ ).

Theorem 3.3. Two strongly invariant Fedosov star-products are equivalent by a strongly invariant equivalence if and only if their characteristic 2-forms lie in the same cohomology class in $H_{\mathfrak{h}}^{G, 2}(M)[[\hbar]]$.

Proof. Let $*_{0}$ and $*_{1}$ two strongly invariant Fedosov star-products with respective characteristic 2 -form $\omega_{\hbar}^{(0)}$ and $\omega_{\hbar}^{(1)}$.

First assume that $\omega_{\hbar}^{(0)}=\omega_{\hbar}^{(1)}+d \alpha$ for some $\alpha=\sum_{k} \hbar^{k} \alpha^{(k)} \in \Omega_{\mathfrak{h}}^{1}(M)^{G}[[\hbar]]$, and define $\omega_{\hbar}(t)=\omega_{\hbar}^{(0)}+t d \alpha$. Let $D_{t}=\partial-\delta+\frac{1}{\hbar}[r(t),-]$ be the Fedosov differential with Weyl curvature $\Omega(t)=\omega+\hbar \omega_{\hbar}(t)$. Let $H(t) \in \Gamma(M, W)$ be the solution of the equation

$$
D_{t} H(t)=-\alpha+\dot{r}(t)
$$

with $\sigma(H(t))=0$. Then $H(t)$ is $G \times H$-invariant since $D_{t}, \alpha$ and $r(t)$ are. According to 9 , Theorem 5.5.3] the solution of the Heisenberg equation

$$
\frac{d F(t)}{d t}+[H(t), F(t)]_{\circ}=0, \quad F(0)=f
$$

establishes an isomorphism $Z_{D_{0}}^{0}(W) \rightarrow Z_{D_{1}}^{0}(W), f \mapsto F(1)$ and then the corresponding series of differential operators $Q:\left(\mathcal{O}_{M}[[\hbar]], *_{0}\right) \rightarrow\left(\mathcal{O}_{M}[[\hbar]], *_{1}\right)$ is obviously $G \times H$-invariant.

Remember from the proof of Proposition 2.5 that in local Darboux coordinates the Fedosov differential writes $D_{t}=d+\frac{1}{\hbar}[\gamma(t),-]_{\circ}$ and $\sigma_{t}^{-1}(h)=h-\iota_{\chi_{h}} \gamma(t)$ if $h \in \mathfrak{h}$. Now remark that $\dot{\gamma}(t)=\dot{r}(t)$ and that $\iota_{\chi_{h}} r(t)$ is independent of $t$. Hence $\sigma_{t}^{-1}(h)$ does not depend on $t$ and thus $Q(h)=h$.

Conversely, assume that $*_{0}$ and $*_{1}$ are related by a strongly invariant equivalence with $\left[\omega_{\hbar}^{(0)}\right] \neq\left[\omega_{\hbar}^{(1)}\right]$ in $H_{\mathfrak{h}}^{G, 2}(M)[[\hbar]]$. Write $\omega_{\hbar}^{(i)}=\sum_{k>0} \hbar^{k-1} \omega_{k}^{(i)}(i=0,1)$ and denote by $l$ the lowest integer for which $\left[\omega_{l}^{(0)}\right] \neq\left[\omega_{l}^{(1)}\right]$ in $H_{\mathfrak{h}}^{G, 2}(M)$. Let us then define

$$
\omega_{\hbar}^{(2)}=\sum_{0<k<l} \hbar^{k-1} \omega_{k}^{(0)}+\sum_{k \geq l} \hbar^{k-1} \omega_{k}^{(1)}
$$

and $*_{2}$ the strongly invariant Fedosov star-product with characteristic 2 -form $\omega_{\hbar}^{(2)}$. Since $\left[\omega_{\hbar}^{(2)}\right]=\left[\omega_{\hbar}^{(1)}\right] \in H_{\mathfrak{h}}^{G, 2}(M)[[\hbar]]$ then $*_{2}$ is equivalent to $*_{1}$, and hence to $*_{0}$, by a strongly invariant equivalence. Then we deduce from Lemma 2.3 that $C_{l+1}^{2}-C_{l+1}^{0}=\pi_{r}^{\#}\left(\omega_{l}^{(1)}-\omega_{l}^{(0)}\right)$, where $C_{k}^{i}$,s $(i=0,2)$ are the cochains defining $*_{i}$. Thus it follows from Proposition 3.1 that $\omega_{l}^{(1)}-\omega_{l}^{(0)}$ is exact and we obtain a contradiction.

Theorem 3.4. Any strongly invariant quantization of $\pi_{r}$ is equivalent to a strongly invariant Fedosov star-product by a strongly invariant equivalence. Therefore the set of strongly invariant star-products quantizing $\pi_{r}$ up to strongly invariant equivalences is an affine space modeled on $H_{\mathfrak{h}}^{G, 2}(M)[[\hbar]]=H_{r}^{2}(U, \mathfrak{g})$. 
Proof. We follow [4, Proposition 4.1].

Let $*$ be an arbitrary strongly invariant quantization of $\pi_{r}$. Denote by $*_{0}$ the strongly invariant Fedosov star-product with the trivial characteristic 2 -form, which coincides with $*$ up to order 0. Moreover, the skew-symmetric part of the first order term in $*-*_{0}$ vanishes, hence it follows from Proposition 3.1 that there exists a strongly invariant equivalence $Q^{(0)}=$ $1+\hbar Q_{0}$ that transforms $*$ into a new strongly invariant quantization $*^{(0)}$ which coincides with $*_{0}$ up to order 1 . Now the skew-symmetric part of the second order term in $*^{(0)}-*_{0}$ yields a closed form $\omega_{1} \in \Omega_{\mathfrak{h}}^{2}(M)^{G}$.

Denote by $*_{1}$ the strongly invariant Fedosov star-product with characteristic 2 -form $\omega_{1}$. Lemma 2.3 tells us that the skew-symmetric part of the second order term in $*^{(0)}-*_{1}$ vanishes, hence it follows from Proposition 3.1 that there exists a strongly invariant equivalence $Q^{(1)}=$ $1+\hbar^{2} Q_{1}$ that transforms $*^{(0)}$ into a new strongly invariant quantization $*^{(1)}$ which coincides with $*_{1}$ up to order 2 .

Repeating this procedure we get a sequence of strongly invariant equivalences $Q^{(k)}=$ $1+\hbar^{k+1} Q_{k}(k \geq 0)$ and a sequence of closed forms $\omega_{k}(k>0)$ such that the strongly invariant quantization $*^{(k)}$ obtained from $*$ by applying successively $Q^{(0)}, \ldots, Q^{(k)}$ coincides up to order $k+1$ with the strongly invariant Fedosov star-product $*_{k}$ with characteristic 2 -form $\omega_{1}+\cdots+\hbar^{k-1} \omega_{k}$.

Finally, the strongly invariant equivalence $Q:=\cdots Q^{(2)} Q^{(1)} Q^{(0)}$ transform $*$ into the strongly invariant Fedosov star-product with characteristic 2-form $\omega_{\hbar}=\sum_{k>0} \hbar^{k-1} \omega_{k}$.

3.3. Classification of dynamical twist quantizations. Let $T$ be a gauge equivalence of dynamical twist quantizations $J_{1}$ and $J_{2}$ of $r$. One can view $T$ as an element in $\operatorname{Diff}(U \times$ $G)^{G \times H}[[\hbar]]$ such that $T(u)=u$ for any $u \in \mathcal{O}_{\mathfrak{h}^{*}}$. Moreover if we denote by $*_{i}^{\prime}$ the compatible quantization of $\pi_{r}$ corresponding to $J_{i}(i=1,2)$ then it follows from an easy calculation that

$$
T\left(f *_{1}^{\prime} g\right)=T(f) *_{2}^{\prime} T(g) \text {. }
$$

Conversely, any $G \times H$-invariant gauge equivalence $T$ from $*_{1}^{\prime}$ to $*_{2}^{\prime}$ which is such that $T(u)=u$ for any $u \in \mathcal{O}_{\mathfrak{h}^{*}}$, that we will call from now a compatible equivalence, obviously gives rise to a gauge equivalence of the dynamical twist quantizations $J_{1}$ and $J_{2}$.

Therefore, the set of dynamical twist quantization of $r$ up to gauge equivalences is in bijection with the set of compatible quantizations of $\pi_{r}$ up to compatible equivalences.

Remember from Proposition 1.7 and Remark 1.8 that any strongly invariant quantization is equivalent to a compatible one by a strongly invariant equivalence. Moreover the PBW star-product has the following nice property: for any $h \in \mathfrak{h}, h^{*_{P B W}}=h^{n}$. Hence any strongly invariant equivalence between two compatible quantizations is actually a compatible equivalence. Consequently:

\section{Proposition 3.5. There is a bijection}

$$
\frac{\left\{\text { strongly invariant quantizations of } \pi_{r}\right\}}{\text { strongly invariant equivalences }} \longleftrightarrow \frac{\left\{\text { compatible quantizations of } \pi_{r}\right\}}{\text { compatible equivalences }}
$$

End of the proof of Theorem 0.2 Assume that the dynamical $r$-matrix is symplectic. Then Theorem 0.2 follows from Proposition 3.5 and Theorem 3.4 .

\section{The QUANTUM COMPOSITION FORMUla}

In this section we assume that $\mathfrak{h}=\mathfrak{t} \oplus \mathfrak{m}$ is a nondegenerate reductive splitting and we denote by $r_{\mathfrak{t}}^{\mathfrak{m}}: \mathfrak{t}^{*} \supset V \rightarrow \wedge^{2} \mathfrak{h}$ the corresponding symplectic dynamical $r$-matrix. Let $p: \mathfrak{h} \rightarrow \mathfrak{t}$ be the $\mathfrak{t}$-invariant projection along $\mathfrak{m}$. For any function $f$ on $\mathfrak{h}^{*}$ with values in a $\mathfrak{h}$-module $L$ we write $f_{\mid \mathfrak{t}^{*}}$ for the function $f \circ p^{*}$ on $\mathfrak{t}^{*}$ with values in $L$ viewed as a $\mathbf{t}$-module; in particular if $f$ is $\mathfrak{h}$-invariant then $f_{\mid \mathfrak{t}^{*}}$ is $\mathbf{t}$-invariant. 
4.1. The classical composition formula (proof of Proposition 0.3). Let $\rho: U \rightarrow \wedge^{2} \mathfrak{g}$ be a dynamical $r$-matrix with $Z \in\left(\wedge^{3} \mathfrak{g}\right)^{\mathfrak{g}}$. Then $\pi:=\pi_{r_{\mathfrak{t}}^{\mathfrak{m}}}+\pi_{\rho}$ defines a $\mathfrak{g}$-quasi-Poisson structure (with the same $Z$ ) on the manifold $X=V \times H \times U \times G$ which is

- $H$-invariant with respect to left multiplication on $H$,

- $H$-invariant with respect to the right action on $U \times G$,

- $T$-invariant with respect to the right action on $V \times H$.

The right diagonal $H$-action, given by $(\tau, x, \lambda, y) \cdot q=\left(\tau, q^{-1} x, \operatorname{Ad}_{q}^{*} \lambda, y q\right)$, actually comes from a momentum map:

$$
\begin{aligned}
\mu: X & \longrightarrow \mathfrak{h}^{*} \\
(\tau, x, \lambda, y) & \longmapsto \lambda-\operatorname{Ad}_{x^{-1}}^{*}\left(p^{*} \tau\right) .
\end{aligned}
$$

Consequently we can apply the reduction with respect to $\mu$. The right $H$-invariant smooth map

$$
\begin{aligned}
\psi: X=V \times H \times U \times G & \longrightarrow M:=U \cap V \times G \\
(\tau, x, \lambda, y) & \longmapsto(\tau, y x)
\end{aligned}
$$

restricts to a diffeomorphism $\mu^{-1}(0) / H \rightarrow M$ with inverse given by

$$
(\tau, y) \longmapsto \overline{\left(\tau, 1, p^{*} \tau, y\right)} \quad(\tau \in U \cap V, y \in G) .
$$

Remark 4.1. From an algebraic viewpoint, we have an injective map of commutative algebras $\psi^{*}: \mathcal{O}_{M} \rightarrow \mathcal{O}_{X}$ with values in $\mathcal{O}_{X}^{\mathfrak{h}}=\mathcal{O}_{X / H}$ and such that, composed with the projection $\mathcal{O}_{X}^{\mathfrak{h}} \rightarrow \mathcal{O}_{X}^{\mathfrak{h}} /\left(\mathcal{O}_{X}^{\mathfrak{h}} \cap<\operatorname{im}\left(\mu^{*}\right)>\right)=\mathcal{O}_{\mu^{-1}(0) / H}$, it becomes an isomorphism.

Since $\psi$ is obviously left $G$-invariant then it remains to show that the induced $\mathfrak{g}$-quasiPoisson structure on $M$ is $\pi_{\rho_{t^{*}}+r_{\mathfrak{t}}^{\mathrm{m}}}$. Let $t, t^{\prime} \in \mathfrak{t} \subset \mathcal{O}_{\mathfrak{t}^{*}}$ and $f, g \in \mathcal{O}_{G}$. First of all we have

$$
\left\{\psi^{*} t, \psi^{*} t^{\prime}\right\}_{X}=\left\{t, t^{\prime}\right\}_{X}=\left[t, t^{\prime}\right]=\psi^{*}\left[t, t^{\prime}\right]
$$

hence $\left\{t, t^{\prime}\right\}_{M}=\left[t, t^{\prime}\right]$. Then

$$
\left\{\psi^{*} t, \psi^{*} f\right\}_{X}=\{t, f(y x)\}_{X}=\vec{t}^{H} \cdot(f(y x))=(\vec{t} \cdot f)(y x)=\psi^{*}(\vec{t} \cdot f) .
$$

The third equality follows from the left $H$-invariance of $\vec{t}^{H}$. Thus $\{t, f\}_{M}=\vec{t} \cdot f$. Finally

$$
\begin{aligned}
\left\{\psi^{*} f, \psi^{*} g\right\}_{X}(\tau, x, \lambda, y) & ={\overrightarrow{r_{\mathfrak{t}}^{\mathfrak{m}}(\tau)}}^{H} \cdot(f(y x), g(y x))+\overrightarrow{\rho(\lambda)}^{G} \cdot(f(y x), g(y x)) \\
& =\left(\overrightarrow{r_{\mathfrak{t}}^{\mathfrak{m}}(\tau)} \cdot(f, g)\right)(y x)+\left(\overrightarrow{\rho\left(\operatorname{Ad}_{x}^{*} \lambda\right)} \cdot(f, g)\right)(y x) .
\end{aligned}
$$

Therefore, when restricting to $\mu^{-1}(0)$ one obtains

$$
\begin{aligned}
\left\{\psi^{*} f, \psi^{*} g\right\}_{X}\left(\tau, x, \operatorname{Ad}_{x^{-1}}^{*}\left(p^{*} \tau\right), y\right) & \left.=\overrightarrow{\left(\overrightarrow{r_{\mathfrak{m}}}(\tau)\right.} \cdot(f, g)\right)(y x)+\left(\overrightarrow{\rho\left(p^{*} \tau\right)} \cdot(f, g)\right)(y x) \\
& \left.=\psi^{*}\left(\overrightarrow{\left(r_{\mathfrak{t}}^{\mathfrak{m}}+\rho_{\mid \mathfrak{t}^{*}}\right.}\right) \cdot(f, g)\right) .
\end{aligned}
$$

Therefore $\{f, g\}_{M}=\overrightarrow{\left(r_{\mathfrak{t}}^{\mathfrak{m}}+\rho_{\mid \mathfrak{t}^{*}}\right)} \cdot(f, g)$. This ends the proof of Proposition 0.3,

4.2. Quantization of the momentum map $\mu$. Let us first consider $\left(V \times H, \pi_{r_{t}^{\mathfrak{m}}}\right)$. There is a momentum map

$$
\begin{aligned}
\nu: V \times H & \longrightarrow \mathfrak{h}^{*} \\
(\tau, x) & \longmapsto-\operatorname{Ad}_{x^{-1}}\left(p^{*} \tau\right)
\end{aligned}
$$

with corresponding right $H$-action on $V \times H$ given by $(\tau, x) \cdot q=\left(\tau, q^{-1} x\right)$.

Like in subsection 2.3 one has a $T$-invariant and $H$-invariant torsion free connexion on $V \times H$, therefore from Proposition 2.5 the corresponding Fedosov star-product $*$ is both strongly $\mathfrak{h}$-invariant and strongly $\mathfrak{t}$-invariant 5 .

\footnotetext{
${ }^{5}$ Remind that we also have a momentum map $V \times H \rightarrow \mathfrak{t}^{*} ;(\tau, x) \mapsto \tau$ with corresponding right $T$-action given by $(\tau, x) \cdot b=\left(\operatorname{Ad}_{b}^{*}(\tau), x b\right)$.
} 
Then Proposition 1.7 tells us that there exists a strongly t-invariant (and $H$-invariant) equivalence $Q$ such that $*^{\prime}:=*^{(Q)}$ is a compatible quantization of $\pi_{r_{\mathrm{t}}^{\mathfrak{m}}}$. Consequently we can define the following algebra morphism:

$$
\mathbf{N}:=Q^{-1} \circ U\left(\nu^{*}\right) \circ \operatorname{sym}:\left(\mathcal{O}_{\mathfrak{h}^{*}}[[\hbar]], *_{P B W}\right) \rightarrow\left(\mathcal{O}_{V \times H}[[\hbar]], *^{\prime}\right) .
$$

It is obviously a quantization of the Poisson map $\nu$ and, moreover, for any $h \in \mathfrak{h}$ and any $f \in \mathcal{O}_{V \times H}$ one has

$$
[\mathbf{N}(h), f]_{*^{\prime}}=Q^{-1}\left(\left[\nu^{*} h, Q(f)\right]_{*}\right)=Q^{-1}\left(\hbar\left\{\nu^{*} h, Q(f)\right\}\right)=\hbar\left\{\nu^{*} h, f\right\} .
$$

In other words, $\mathbf{N}$ is a quantum momentum map quantizing $\nu$.

Let us now assume that we know a dynamical twist quantization $J(\lambda): U \rightarrow \otimes^{2} U \mathfrak{g}[[\hbar]]$ of $\rho(\lambda)$ (with some associator $\Phi$ ) and denote by $*_{J}$ the corresponding compatible quantization of $\pi_{\rho}$ on $U \times G$. Together with $*^{\prime}$ it induces a quantization $*_{J}^{\prime}$ of $\pi_{r_{\mathrm{t}}^{\mathrm{m}}}+\pi_{\rho}$ on $X$ (with the same $\Phi)$.

Remark 4.2. Actually $*_{J}^{\prime}$ is the compatible quantization corresponding to the dynamical twist quantization $\mathbf{J}(\tau, \lambda):=J_{\mathfrak{t}}^{\mathfrak{m}}(\tau) J(\lambda):(\mathfrak{t} \oplus \mathfrak{h})^{*} \supset V \times U \longrightarrow \otimes^{2} U(\mathfrak{h} \oplus \mathfrak{g})[[\hbar]]$ of the dynamical $r$-matrix $\mathbf{r}(\tau, \lambda):=r_{\mathfrak{t}}^{\mathfrak{m}}(\tau)+\rho(\lambda): V \times U \longrightarrow \wedge^{2}(\mathfrak{h} \oplus \mathfrak{g})$. Here $J_{\mathfrak{t}}^{\mathfrak{m}}$ is the dynamical twist quantizing $r_{\mathfrak{t}}^{\mathfrak{m}}$.

For any $f \in \mathcal{O}_{\mathfrak{h}^{*}}$ we define $\mathbf{M}(f):=(\mathbf{N} \otimes$ inc $) \circ \Delta(f) \in\left(\mathcal{O}_{V \times H} \otimes \mathcal{O}_{U \times G}\right)[[\hbar]]=\mathcal{O}_{X}[[\hbar]]$. Here inc : $\mathcal{O}_{\mathfrak{h}^{*}} \hookrightarrow \mathcal{O}_{U \times G}$ is the natural inclusion and $\Delta: \mathcal{O}_{\mathfrak{h}^{*}} \rightarrow \mathcal{O}_{\mathfrak{h}^{*}} \otimes \mathcal{O}_{\mathfrak{h}^{*}}=\mathcal{O}_{\mathfrak{h}^{*} \times \mathfrak{h}^{*}}$ is defined by $\Delta(f)\left(\lambda_{1}, \lambda_{2}\right)=f\left(\lambda_{1}+\lambda_{2}\right)$.

Proposition 4.3. The algebra morphism

$$
\mathbf{M}:\left(\mathcal{O}_{\mathfrak{h}}[[\hbar]], *_{P B W}\right) \longrightarrow\left(\mathcal{O}_{X}[[\hbar]], *_{J}^{\prime}\right)
$$

is a quantum momentum map quantizing $\mu$.

4.3. Quantization of the composition formula (proof of Theorem 0.4). Let us assume that $J$ is a dynamical twist quantization of $\rho$ and keep the notations of the previous subsection.

Denote by $\mathcal{I}$ the right ideal generated by $\operatorname{im}(\mathbf{M})$ in $\left(\mathcal{O}_{X}[[\hbar]], *_{J}^{\prime}\right)$ and consider the reduced algebra $\mathcal{A}:=\mathcal{O}_{X}^{\mathfrak{h}}[[\hbar]] / \mathcal{O}_{X}^{\mathfrak{h}}[[\hbar]] \cap \mathcal{I}$. Let $\Psi=\psi^{*}+O(\hbar)$ be the composition of $\psi^{*}: \mathcal{O}_{M}[[\hbar]] \rightarrow$ $\mathcal{O}_{X}^{\mathfrak{h}}[[\hbar]]$ with the projection $\mathcal{O}_{X}^{\mathfrak{h}}[[\hbar]] \rightarrow \mathcal{A} \cong \mathcal{O}_{\mu^{-1}(0) / H}[[\hbar]]$. It is obviously bijective and $G$-invariant (since $\psi^{*}$ is), therefore it defines a quantization $\tilde{*}$ of the quasi-Poisson structure $\pi_{r_{\mathfrak{t}}^{\mathfrak{m}}+\rho_{\mid t^{*}}}$. We end the proof of Theorem 0.4 using the following proposition:

Proposition 4.4. $\tilde{*}$ is a compatibe quantization.

Proof. First of all for any $u, v \in \mathcal{O}_{\mathrm{t}^{*}}$ one has

$$
\left(\psi^{*} u\right) *_{J}^{\prime}\left(\psi^{*} v\right)=u *_{J}^{\prime} v=u *_{P B W} v=\psi^{*}\left(u *_{P B W} v\right) .
$$

Consequently $u \tilde{*} v=\Psi^{-1}(\Psi(u) \cdot \mathcal{A} \Psi(v))=u *_{P B W} v$.

Then let $u \in \mathcal{O}_{\mathrm{t}^{*}}$ and $f \in \mathcal{O}_{G}$. On one hand

$$
\left(\psi^{*} f\right) *_{J}^{\prime} \psi^{*} u=(f(y x)) *_{J}^{\prime} u=f(y x) u=\psi^{*}(f u)
$$

and thus $f \tilde{*} u=f u$. On the other hand for $u=t^{n}(t \in \mathfrak{t})$ one has

$$
\begin{gathered}
\psi^{*}\left(t^{n}\right) *_{J}^{\prime}\left(\psi^{*} f\right)=\left(t^{n}\right) *_{J}^{\prime}(f(y x))=\sum_{k=0}^{n} \hbar^{k} C_{n}^{k}\left(\left(\vec{t}^{H}\right)^{k} \cdot(f(y x))\right) t^{n-k} \\
=\sum_{k=0}^{n} \hbar^{k} C_{n}^{k}\left(\vec{t}^{k} \cdot f\right)(y x) t^{n-k}=\psi^{*}\left(\sum_{k=0}^{n} \hbar^{k} C_{n}^{k}\left(\vec{t}^{k} \cdot f\right) t^{n-k}\right) .
\end{gathered}
$$

Therefore $t^{n} \tilde{*} f=\sum_{k=0}^{n} \hbar^{k} C_{n}^{k}\left(\vec{t}^{k} \cdot f\right) t^{n-k}$. The proposition is proved. 


\section{REFERENCES}

[1] A. Alekseev and Y. Kosmann-Schwarzbach, Manin pairs and momentum maps, J. Diff. geom. 56 (200), no. $1,133-165$.

[2] A. Alekseev, Y. Kosmann-Schwarzbach and E. Meinrenken, Quasi-Poisson manifolds, Canad. J. Math. 54 (2002), no. 1, 3-29.

[3] M. Bertelson, P. Bielavsky and S. Gutt, Parametrizing equivalence classes of invariant star-products, Lett. Math. Phys. 46 (1998), 339-345.

[4] M. Bertelson, M. Cahen and S. Gutt, Equivalence of star-products, Classical Quantum Gravity 14 (1997), A93-A107.

[5] V. Drinfeld, Quasi-Hopf algebras, Leningrad Math. J. 1 (1990), 1419-1457.

[6] B. Enriquez and P. Etingof, Quantization of Alekseev-Meinrenken dynamical $r$-matrices, Trans. Am. Math. Soc. (ser. 2) 210 (2003), 81-98.

[7] B. Enriquez and P. Etingof, Quantization of classical dynamical $r$-matrices with nonabelian base, Comm. Math. Phys. 254 (2005), no. 3, 603-650.

[8] B. Fedosov, A simple geometric construction of deformation quantization, J. of Diff. Geom. 40 (1994), 213-238.

[9] B. Fedosov, Deformation quantization and index theory, Akademia Verlag, Berlin, 1996.

[10] B. Fedosov, Nonabelian reduction in deformation quantization, Lett. Math. Phys. 43 (1998), 137-154.

[11] L. Fehér, A. Gábor and P. Pusztai, On dynamical $r$-matrices obtained from Dirac reduction and their generalizations to affine Lie algebras, J. Phys. A34 (2001), no. 36, 7335-7348.

[12] O. Kravchenko, Deformation quantization of symplectic fibrations, Compos. Math. 123 (2000), no. 2, 131-265.

[13] P. Xu, Triangular dynamical $r$-matrices and quantization, Adv. Math. 166 (2002), no. 1, 1-49.

[14] P. Xu, Quantum dynamical Yang-Baxter equation over a nonabelian base, Comm. Math. Phys. 226 (2002), no. 3, 475-495. 